\title{
Intersections
}

Canadian Journal of Music

Revue canadienne de musique

\section{Jean-Christophe Branger et Malou Haine. 2014. Ernest van Dyck et Jules Massenet : Un interprète au service d'un compositeur. Paris : VRIN, 172 p. Coll. " MusicologieS ». ISBN : 978-2-7116-2547}

\section{Frédéric Lemmers}

Volume 36, numéro 1, 2016

URI : https://id.erudit.org/iderudit/1043870ar

DOI : https://doi.org/10.7202/1043870ar

Aller au sommaire du numéro

Éditeur(s)

Canadian University Music Society / Société de musique des universités canadiennes

ISSN

1911-0146 (imprimé)

1918-512X (numérique)

Découvrir la revue

Citer ce compte rendu

Lemmers, F. (2016). Compte rendu de [Jean-Christophe Branger et Malou Haine. 2014. Ernest van Dyck et Jules Massenet : Un interprète au service d'un compositeur. Paris : VRIN, 172 p. Coll. « MusicologieS ». ISBN : 978-2-7116-2547]. Intersections, 36(1), 91-94. https://doi.org/10.7202/1043870ar

Copyright @ Canadian University Music Society / Société de musique des universités canadiennes, 2018
Ce document est protégé par la loi sur le droit d'auteur. L'utilisation des services d'Érudit (y compris la reproduction) est assujettie à sa politique d'utilisation que vous pouvez consulter en ligne.

https://apropos.erudit.org/fr/usagers/politique-dutilisation/ 
Jean-Christophe Branger et Malou Haine. 2014. Ernest van Dyck et Jules Massenet: Un interprète au service d'un compositeur. Paris: VRIN, 172 p. Coll. «MusicologieS». ISBN : 978-2-7116-2547.

Le ténor belge Ernest Van Dyck (1861-1923) est principalement connu pour avoir été une figure emblématique du chant wagnérien, en particulier au festival de Bayreuth où il se produisit régulièrement dans le rôle-titre de Parsifal durant plus d'une décennie (1888-1901), mais aussi à Paris où il se fit connaître du monde francophone en défendant le répertoire wagnérien aux Concerts Lamoureux (1883-1893). Cependant, de cette brillante carrière internationale dominée par le chant wagnérien, les notices biographiques existantes et les autres ouvrages évoquant le parcours de Van Dyck retiennent aussi la défense d'un certain répertoire français, en particulier deux opéras de Jules Massenet (1842-1912): Manon, dont Van Dyck tenait le rôle de Des Grieux, et Werther, dont il créa le rôle-titre le 16 février 1892 à l'Opéra de Vienne, en allemand, dans une traduction de l'auteur, critique et traducteur viennois Max Kalbeck (1850-1921).

C'est de la genèse de cette création dont il est principalement question dans l'ouvrage de Jean-Christophe Branger et de Malou Haine qui entendent y apporter un éclairage nouveau au départ de sources inédites conservées dans les archives familiales de la famille Van Dyck. Ils s'appuient en particulier sur une sélection de 130 lettres $^{1}$ - illustrant le caractère amical de la collaboration artistique entretenue par Van Dyck et Massenet - annotées, commentées, remises dans leur contexte et accompagnées d'une riche iconographie.

Cette correspondance, qui reste concentrée dans le temps en ne s'étalant pas beaucoup plus largement que sur une décennie (1890-1903), et l'appareil critique qui l'accompagne, précisent les circonstances de la création de Werther ainsi que le rôle effectif de Van Dyck dans les préparatifs de cet événement, à savoir qu'il fut l'orchestrateur du processus de traduction du livret original. Dans cet ouvrage, Branger et Haine mettent en évidence le caractère singulier du contexte de la création de Werther par rapport à celui dans lequel Massenet avait l'habitude de faire créer ses œuvres. On y apprend notamment que, contrairement à ses habitudes, Massenet ne semblait pas avoir un nom d'artiste en tête pour le rôle-titre de Werther au moment de sa composition, et que le choix de Vienne est à rechercher ailleurs que dans la volonté du compositeur de recourir aux services de l'ami talentueux qu'il avait alors là-bas. Le choix des documents repris dans cet ouvrage et leur commentaire critique suggèrent plutôt l'hypothèse d'une conjonction d'éléments ayant fait de Vienne une

1 Une dizaine de ces lettres ne proviennent pas de ce corpus familial. 
opportunité pour Massenet plus qu'un choix délibéré. En effet, pour le compositeur, les perspectives de voir créer son œuvre à Paris à la fin des années 1880 étaient devenues faibles, voire nulles, depuis que le directeur de l'OpéraComique, Léon Carvalho (1825-1897), avait refusé de la programmer pour l'avoir jugée trop triste, et que la Salle Favart avait été détruite par un incendie en 1887. Branger et Haine précisent aussi la nature et la chronologie de l'amitié ayant existé entre les deux hommes en montrant, d'une part, qu'elle naquit à Vienne au moment de la création allemande de Manon à l'Opéra impérial, soit en 1890 et, d'autre part, qu'elle fut principalement alimentée par leur collaboration artistique viennoise. De la création allemande de Manon, on apprend que le succès fut au rendez-vous en dépit d'une mise en scène «à l'économie», et que cela valut à Massenet de se voir offrir de nouvelles propositions de la part du directeur de l'Opéra de Vienne, Whilhelm Jahn (1835-1900). Bien entendu, le fait que Van Dyck venait de connaître un succès personnel très important lors de cette création ne gâchait rien à l'affaire. Tous les ingrédients étaient donc réunis pour envisager la création mondiale de Werther à Vienne, avec l'une des plus grandes figures du chant wagnérien de son temps dans le rôletitre. De là, l'aide de Van Dyck dans le processus de traduction en allemand de l'œuvre à créer.

L'exposé introductif d'une trentaine de pages intitulé «Les relations entre Van Dyck et Massenet: des liens amicaux ou professionnels?» et la sélection de lettres qui s'ensuit montrent clairement que la collaboration viennoise entre les deux artistes reste un phénomène isolé dans leurs parcours respectifs. Extraits de presse à l'appui et morceaux choisis dans les souvenirs personnels de Van Dyck, les auteurs montrent que Van Dyck n'intègre pas le premier cercle des proches du compositeur. Werther ne lui sera d'ailleurs pas dédié et Massenet ne composera jamais aucun opéra pour lui, alors qu'il le fit pour d'autres.

Une synthèse des débuts de Van Dyck retrace le parcours du jeune anversois, de ses études de droit dans sa Belgique natale à son voyage de noces à Bayreuth au lendemain de son mariage avec Augusta Servais (1860-1925), la fille du compositeur et chef d'orchestre belge Franz Servais (1846-1901). On le voit monter à Paris où il devint correspondant au journal belge L'Escaut. On le suit dans ses premiers succès aux Concerts Lamoureux, au début des années 1880, et l'on s'aperçoit que c'est à cette époque que remonte sa première rencontre avec Massenet; elle ne fut pas suivie d'une collaboration artistique régulière. Cela permet de mieux saisir le parcours de celui qui, doté d'une forte personnalité et d'un goût musical affûté, faisait partie de ces quelques artistes qui, déjà à la fin $\mathrm{du} \mathrm{XIX}^{\mathrm{e}}$ siècle, estimaient devoir chanter l'opéra en langue originale alors que la pratique dominante était de chanter soit le répertoire national soit en traduction. Critique musical à ses débuts, Van Dyck ne manquait pas de s'exprimer sur la qualité des créations lyriques de son temps : s'il appréciait l'œuvre de Massenet, il ne lui vouait pas la même admiration qu'à Wagner et, de la scène parisienne, il semblait lui préférer un Chabrier.

Par ailleurs, le corpus de lettres publié dans cet ouvrage illustre un autre aspect de la collaboration artistique entre Van Dyck et Massenet à l'époque de la création de Werther : celui de la composition d'une musique de ballet 
sur un livret intitulé Carillon, légende mimée et dansée, écrit conjointement par Camille De Roddaz (1846-1896) et son ami Van Dyck. En juxtaposant les correspondances entre Van Dyck et de Roddaz, et celle entre le grand ténor et le compositeur français, Branger et Haine mettent en exergue l'existence d'un troisième homme venu s'immiscer dans la collaboration artistique entre les deux grands artistes. De cette correspondance, on perçoit bien l'empressement de Camille de Roddaz de se voir aider par son cher ami Van Dyck dans l'établissement d'une collaboration avec le chantre de l'opéra français de cette fin de siècle.

En définitive, le corpus de lettres publié dans cet ouvrage reste incomplet. Essentiellement constitué de lettres adressées à Van Dyck, il en manque les envois ou les réponses, suivant le cas. Celles-ci ne sont pas localisées à l'heure actuelle. Bien que couvrant une période s'étalant de l'automne 1890 à l'année 1912, ce corpus de lettres est principalement concentré sur les années 18901892. Trois quarts des lettres se rapportent en effet aux créations viennoises de Manon et de Werther. Le reste se rapporte à la création du Carillon, à la création en langue allemande de La Navarraise (1895) et aux représentations parisiennes de Van Dyck dans Werther en 1903, à l'Opéra-Comique, désormais dirigé par Albert Carré (1852-1938).

En conclusion, cet ouvrage s'adresse surtout à ceux qui s'intéressent à la vie lyrique de la fin du XIX ${ }^{\mathrm{e}}$ siècle, que ce soit l'opéra-comique français en général, l'œuvre de Massenet en particulier ou les grands interprètes de cette époque. Il ravira ceux qui sont intrigués par les coulisses de la création et de la programmation des grandes scènes lyriques de cette époque. Quant aux wagnériens, ils y trouveront un éclairage nouveau sur les circonstances qui ont conduit l'une des grandes figures de leur scène fétiche, Bayreuth, à se voir associée à l'histoire du répertoire d'opéra-comique français.

FRÉDÉRIC LEMMERS

\section{BIOGRAPHIE}

Frédéric Lemmers est licencié en musicologie de l'Université Libre de Bruxelles. Après avoir travaillé plusieurs années comme chercheur à la section de la musique de la Bibliothèque royale de Belgique, il est depuis 2011 responsable de la politique de numérisation de cette institution. Dans ce cadre, il a dirigé de nombreux projets de numérisation patrimoniale menés aux niveaux belge et européen se rapportant entre autres au patrimoine musical. Il dirige actuellement un important projet de numérisation des fonds de musique enregistrée sur disques 78 tours conservés à la Bibliothèque royale de Belgique. Très actif au plan international au sein d'associations professionnelles de bibliothèques patrimoniales, Frédéric Lemmers préside actuellement la section Cataloguing \& Metadata de l'association internationale des Bibliothèques musicales (IAML). Il est également membre du comité de coordination du Consortium of European Research Libraries (CERL) et secrétaire du Réseau Francophone Numérique (RFN). En Belgique, il est membre du Comité UNESCO pour le programme «Mémoire du monde». Ses principales publications se rapportent à la vie 
lyrique belge des années 1880-1950. Administrateur du label discographique « Musique en Wallonie», dont il dirige la collection «historiques», il a publié des rééditions phonographiques d'enregistrements historiques de grandes figures belges de l'art lyrique (Ernest Tilkin Servais, Louis Richard, Fernand Faniard, Lucienne Delvaux, Huberte Vecray). En 2006, il fut commissaire de l'exposition «Opera.be» organisée par la Fondation Roi Baudouin à cette occasion, il a publié un coffret discographique éponyme d'enregistrements historiques conservés au sein de la collection Yves Becko de la Bibliothèque royale de Belgique. Professeur invité à la Haute École Libre de Bruxelles, il y enseigne dans le cadre du Master en Gestion Globale du Numérique. 